\title{
The impact of sulfur precipitation in developing a sour gas reservoir with pressure-sensitive effects
}

\author{
Zhixing $\mathrm{Ru}$, Kang An, Jinghong $\mathrm{Hu}^{\circledR} *$ \\ Beijing Key Laboratory of Unconventional Natural Gas Geology Evaluation and Development Engineering, \\ School of Energy Resources, China University of Geosciences, Beijing 100083, P. R. China \\ (Received May 30, 2019; revised June 12, 2019; accepted June 19, 2019; available online June 22, 2019)
}

\section{Citation:}

Ru, Z., An, K., Hu, J. The impact of sulfur precipitation in developing a sour gas reservoir with pressure-sensitive effects. Advances in Geo-Energy

Research, 2019, 3(3): 268-276, doi: 10.26804/ager.2019.03.05.

Corresponding author:

*E-mail: hujinghong@cugb.edu.cn

\section{Keywords:}

High-sulfur gas

elemental sulfur solubility

sulfur deposition

pressure-sensitive effects

mathematical model

\begin{abstract}
:
During the development of high sulfur gas fields, gaseous sulfur is likely to precipitate and deposit in the reservoirs due to the changes of temperature, pressure, and gas compositions. Therefore, how to establish an accurate prediction model of elemental sulfur solubility in gas mixtures is a key issue. At present, most scholars use Roberts elemental sulfur solubility model (SPE Reserv. Eng. 1997, 12(2): 118-123) to describe the damage caused by sulfur deposition in high-sulfur gas reservoirs. However, some scholars believe that the Roberts model needs to be improved and relevant works have been done. In this study, a one-dimensional radial production model is established using the HU model (J. Nat. Gas Sci. Eng. 2014, 18: 31-38) and the Roberts elemental sulfur solubility model. These models can be used to describe the permeability and pressure changes caused by sulfur deposition more accurately. The results show that the permeability and pressure changes in the Roberts model are larger than that of which in the HU model and the pressure-sensitive effects may increase the reservoir damage. The comparison of the calculated results with the true values shows that the HU model is more accurate. This paper may change a number of views about sulfur deposition in high-sulfur gas reservoirs.
\end{abstract}

\section{Introduction}

High-sulfur gas is a type of special natural gas with hydrogen sulfide compounds, especially hydrogen sulfide (Clark et al., 1989; Karan et al., 1998). In recent years, high-sulfur gas has accumulated in geological reserves up to nearly $7 \times 10^{11}$ $\mathrm{m}^{3}$, accounting for $1 / 8$ of the total domestic natural gas proven reserves. Due to the toxic and corrosive properties of sulfur and the sulfur precipitation in high-sulfur gas reservoirs, the problems associated with high-sulfur gas reservoirs development are much more complex (Li et al., 2015; Santos et al., 2016; Guo et al., 2017).

Trial mining was conducted in the Zhaolan Village highsulfur gas reservoirs in the North China Oil Field in 1976. Because of the lack of theoretical understanding of highsulfur gas reservoir development, the reservoirs were forced to close because of severe sulfur deposition. The wells remain closed until now (Yang, 2001). Solid sulfur may cause the formation of well-bore plug and leads a sharp decline in gas production (Zeng et al., 2005; Cen et al., 2007). Compared with conventional gas reservoirs, it is much more difficult to develop high-sulfur gas reservoirs efficiently (Wang et al., 2011; Fan et al., 2013; Hu et al., 2017a). Puguang gas field, the largest high-sulfur gas field in China, also has the problem that the permeability decreases rapidly in the process of development. It brings adverse effects to production (Zeng et al., 2019). Therefore, it is of great significance to research on the sulfur deposition with temperature and pressure changes, it is useful to understand the laws of sulfur deposition and minimize the effects of sulfur deposition on permeability and pressure.

Many researchers have analyzed the sulfur deposition mechanism and established a Darcy flow percolation model based on the stable gas percolation theory. However, they did not consider the effect of pressure drop on the sulfur deposition and changes of porosity and permeability (Chrastil, 1982; Roberts, 1996; Wang, 1999; Sun and Chen, 2003; Pirzadeh et al., 2014). Guo et al. (2009) did the research on the sulfur deposition in sour gas reservoir, and the optimum productivity of sour gas wells is obtained by a scanning 
electron microscope (SEM) and X-ray mapping. The pressuresensitive effects are not considered in most of their studies as well (Hu et al., 2014). Some other researchers analyzed the influence of pressure on the fluid density changes and obtained the relationship between sulfide solubility and pressure in the reservoir fluid through many experiments (Hu et al., 2014, 2017b; Zhao et al., 2015; Bian et al., 2016; Guo and Wang, 2016; He et al., 2016; Mao et al., 2016; Ou et al., 2016; Ren et al., 2016; Sun et al., 2016; Roberts, 2017; Li et al., 2018). Other researchers also used mathematical methods, such as Grey Correlation Method, to predict the sulfur solubility (Bian et al., 2018).

Based on these studies above, further research has been completed in this work for the establishment of sulfur deposition and permeability model of a gas well in high-sulfur gas reservoirs, which is under the condition of a closed outer boundary and definite production inner boundary. The variation laws of permeability, porosity and formation pressure were obtained with the consideration of sulfur precipitation and reservoir pressure-sensitive effects.

\section{Mechanism of sulfur precipitation}

In the original condition, elemental sulfur and hydrogen are always combined to generate many types of hydrogen sulfide compounds. With the changes in temperature and pressure, hydrogen sulfide compounds can be converted to solid sulfur. The chemical equation is:

$$
\mathrm{H}_{2} \mathrm{~S}_{x+1} \Leftrightarrow \mathrm{H}_{2} \mathrm{~S}+x \mathrm{~S} \downarrow
$$

With the decrease of temperature and pressure, more solid sulfur appears. And the sulfur precipitation may occur in the pore throats, it could even block the fluid flow passage. The permeability decrease rapidly.

The solubility of hydrogen sulfide compounds in highsulfur gas is affected by the changes in temperature and pressure; therefore, it is sensible to study the relationship between them. Roberts (1997) presented a formula to describe the solubility of sulfur in high-sulfur gas based on the model proposed by Chrastil (1982):

$$
c_{s}=\rho^{4} \exp \left(\frac{-4666}{T}-4.5711\right)
$$

where $c_{s}$ is the solubility of sulfur in high-sulfur gas, $\mathrm{kg} / \mathrm{m}^{3}$; and $\rho$ is the fluid density in the reservoirs, $\mathrm{kg} / \mathrm{m}^{3}$. The formula has been verified and a new model to describe the relationship between the solubility of sulfur and fluid density was provided by $\mathrm{Hu}$ et al. (2014) as:

$$
c_{\mathrm{s}}=\rho^{a} \exp \left(\frac{-b}{T}-c\right)
$$

where $a, b$, and $c$ are parameters related to the fluid density. These values could be found in $\mathrm{Hu}$ et al. (2014) in different situations.

It can be obtained by the gas state equation that:

$$
\rho=\frac{M_{a} \gamma_{g} p}{Z R T}
$$

where $M_{a}$ is the molecular weight of air, $28.97 \mathrm{~g} / \mathrm{mol} ; p$ is the formation pressure, MPa; $\gamma_{g}$ is the relative density of the gas; $Z$ is the deviation factor; $T$ is the temperature, $\mathrm{K}$; and $R$ is the universal gas constant, $8.315 \mathrm{~J} /(\mathrm{mol} \cdot \mathrm{K})$.

Combining Eqs. (3) and (4) yields:

$$
\frac{d c_{s}}{d p}=a\left(3.484 \times 10^{3} \frac{\gamma_{g}}{Z T}\right)^{a} \exp \left(\frac{-b}{T}-c\right) p^{a-1}
$$

\section{Establishment and the solution of sulfur de- position model}

The solubility of sulfur decreases with the decline of pressure and temperature in high-sulfur gas reservoirs. Sulfur deposition is especially serious in the region near the wellbore. Therefore, it is important to establish a scientific model of sulfur deposition.

\subsection{Permeability variation in high-sulfur gas reser- voirs}

The sulfur concentration in the reservoir fluid decreases with the pressure reduces. The value of concentration can be easily obtained as:

$$
\Delta c_{s i}=\left(3.484 \times 10^{3} \frac{\gamma_{g}}{Z T}\right)^{a} \exp \left(\frac{-b}{T}-c\right)\left(p_{e}{ }^{a}-p_{i}{ }^{a}\right)
$$

where $\Delta c_{s i}$ is the difference between the current and the initial elemental sulfur solubility, $\mathrm{kg} / \mathrm{m}^{3}$.

Porosity of reservoirs rock would reduce due to the sulfur deposition. The porosity is obtained by its definition as:

$$
\phi_{i}=\frac{V_{i} \phi-V_{i s}}{V_{i}}=\frac{V_{i} \phi-\frac{V_{i} \phi \times \Delta c_{s i}}{\rho_{s}}}{V_{i}}=\phi\left(1-\frac{\Delta c_{s i}}{\rho_{s}}\right)
$$

And:

$$
V_{i}=\pi\left(r_{i+1}^{2}-r_{i}^{2}\right)
$$

where $\phi_{i}$ is the current porosity in radial $i$-th grid; $\phi$ is the initial porosity; $V_{i}$ is the rock volume in radial $i$-th grid, $\mathrm{m}^{3}$; $V_{i s}$ is the volume of solid sulfur in radial $i$-th grid, $\mathrm{m}^{3} ; \rho_{s}$ is the density of solid sulfur, $\mathrm{kg} / \mathrm{m}^{3} ; r_{i}$ is the length in radial $i$-th grid, m; and $r_{i+1}$ is the length in radial $i+1$-th grid, $\mathrm{m}$.

According to Roberts (1997), Zeng et al. (2005) and Pirzadeh et al. (2014), the permeability empirical formula can be obtained with the consideration of sulfur deposition as:

$$
K_{i}=K \exp \left[-6.22 \times\left(1-\frac{\phi_{i}}{\phi}\right)\right]
$$

Combining Eqs. (8) and (9), we have:

$K_{i}=K \exp \left[\frac{-6.22 \times\left(3.484 \times 10^{3} \frac{\gamma_{g}}{Z T}\right)^{a} \exp \left(\frac{-b}{T}-c\right)\left(p_{e}^{a}-p_{i}^{a}\right)}{\rho_{s}}\right]$

When the pressure-sensitive effects is considered: 


$$
K_{i}=K \exp \left[-6.22 \times\left(3.484 \times 10^{3} \frac{\gamma_{g}}{Z T}\right)^{a} \times \frac{\exp \left[-\alpha\left(p_{e}-p_{i}\right)\right] \exp \left(\frac{-b}{T}-c\right)\left(p_{e}^{a}-p_{i}^{a}\right)}{\rho_{s}}\right]
$$

\subsection{Establishment of the mathematical model of a} sour gas well

\subsubsection{Basic equations}

Gas control equation:

$$
\begin{gathered}
\frac{1}{r} \frac{\partial}{\partial r}\left(r \frac{\partial p}{\partial r}\right)=\frac{\phi \mu c}{86.4 K_{\mathrm{i}}} \frac{\partial p}{\partial t} \\
p(r, 0)=p_{i}, r_{w} \leq r \leq r_{e}
\end{gathered}
$$

Closed outer boundary:

$$
\left.\frac{\partial p}{\partial r}\right|_{r=r_{e}}=0,(t>0)
$$

Internal boundary of fixed production:

$$
86.4 \times\left.\frac{2 \pi K_{\mathrm{i}} h r}{\mu} \frac{\partial p}{\partial r}\right|_{r=r_{w}}=q,(t>0)
$$

where $c$ is the coefficient of compressibility, $\mathrm{MPa}^{-1} ; \mu$ is the fluid viscosity, $\mathrm{mPa} \cdot \mathrm{s} ; t$ is time, $\mathrm{d} ; q$ is gas production, $\mathrm{m}^{3} / \mathrm{d}$; and $\psi$ is the pseudo pressure, MPa.

According to the given data and initial boundary conditions, the model can be solved. An actual gas radial flow model could also be obtained when the pressure value is replaced with the pseudo pressure.

\subsubsection{In-homogeneous grid difference equation in a column coordinate system}

In a polar coordinate system, the point center difference equation of the radial in-homogeneous grid is:

$$
\begin{aligned}
\left.\frac{1}{r} \frac{\partial}{\partial r}\left(r \frac{\partial \psi}{\partial r}\right)\right|_{r=r_{i}} & =\frac{1}{r_{i}} \frac{\left.r \frac{\partial \psi}{\partial r}\right|_{r+0.5}-\left.r \frac{\partial \psi}{\partial r}\right|_{r-0.5}}{\Delta r_{i}} \\
& =\frac{1}{r_{i}} \frac{r_{i+0.5} \frac{\psi_{i+1}-\psi_{i}}{\Delta r_{i+0.5}}-r_{i-0.5} \frac{\psi_{i}-\psi_{i-1}}{\Delta r_{i-0.5}}}{\Delta r_{i}}
\end{aligned}
$$

Eq. (13) can be changed into:

$$
\begin{aligned}
\frac{\phi \mu c}{K} \frac{\partial p}{\partial t} & =\frac{r_{i+0.5}}{r_{i}} \frac{\psi_{i+1}}{\Delta r_{i} \Delta r_{i+0.5}}+\frac{r_{i-0.5}}{r_{i}} \frac{\psi_{i-1}}{r_{i} \Delta r_{i-0.5}} \\
& -\left(\frac{r_{i+0.5}}{\Delta r_{i+0.5}}+\frac{r_{i-0.5}}{\Delta r_{i-0.5}}\right) \frac{\psi_{i}}{r_{i} \Delta r_{i}}
\end{aligned}
$$

In a condition of the center of the block grid, Eq. (15) could be obtained from Eq. (13):

$$
\begin{aligned}
\left.\frac{1}{r} \frac{\partial}{\partial r}\left(r \frac{\partial \psi}{\partial r}\right)\right|_{r=r_{i}} & =\frac{1}{r_{i} \Delta r_{i}}\left[\frac{r_{i+0.5}\left(\psi_{i+1}-\psi_{i}\right)}{0.5\left(\Delta r_{i+1}+\Delta r_{i}\right)}\right] \\
& -\frac{1}{r_{i} \Delta r_{i}}\left[\frac{r_{i-0.5}\left(\psi_{i}-\psi_{i-1}\right)}{0.5\left(\Delta r_{i-1}+\Delta r_{i}\right)}\right]
\end{aligned}
$$

Convert the polar coordinate system to a Cartesian coordinate system:

$$
r=r_{w} e^{x},\left(r_{i}=r_{w} e^{x_{i}}\right)
$$

Combining Eqs. (16) and (12), then we can get:

$$
\frac{\partial^{2} \psi}{\partial x^{2}}=e^{2 x} r_{w}^{2} \frac{\phi \mu c}{86.4 K_{i}} \frac{\partial \psi}{\partial t}
$$

Combining $\left.\frac{\partial^{2} \psi}{\partial x^{2}}\right|_{x=x_{i}}=\frac{\psi_{i+1}}{\Delta x_{i} \Delta x_{i+0.5}}+\frac{\psi_{i-1}}{\Delta x_{i} \Delta x_{i-0.5}}-\left(\frac{1}{\Delta x_{i+0.5}}+\right.$ $\left.\frac{1}{\Delta x_{i-0.5}}\right) \frac{\psi_{i}}{\Delta x_{i}}$ with Eq. (17):

$$
\begin{aligned}
& \frac{\psi_{i+1}^{n+1}}{\Delta x_{i} \Delta x_{i+0.5}}-\left(\frac{1}{\Delta x_{i+0.5}}+\frac{1}{\Delta x_{i-0.5}}\right) \frac{\psi_{i}^{n+1}}{\Delta x_{i}}+\frac{\psi_{i-1}^{n+1}}{x_{i} \Delta x_{i-0.5}} \\
& =e^{2 i \Delta x} r_{w}^{2} \frac{\phi \mu c}{86.4 K_{i}} \frac{\psi_{i}^{n+1}-\psi_{i}^{n}}{\Delta t}
\end{aligned}
$$

Using the equidistant grid $\Delta x$, then:

$$
\frac{\psi_{i+1}^{n+1}-2 \psi_{i}^{n+1}+\psi_{i-1}^{n+1}}{\Delta x_{i}^{2}}=e^{2 i \Delta x} r_{w}^{2} \frac{\phi \mu c}{K_{i}} \frac{\psi_{i}^{n+1}-\psi_{i}^{n}}{\Delta t}
$$

Calculating $M(p)=e^{2 i \Delta x} r_{w}^{2} \frac{\phi \mu c}{86.4 K_{i}} \frac{\Delta x^{2}}{\Delta t}$, Eq. (19) can be written as:

$$
\psi_{i+1}^{n+1}-(2+M) \psi_{i}^{n+1}+\psi_{i-1}^{n+1}=-M \psi_{i}^{n}
$$

Then, $\lambda=2+M, d_{i}=-M p_{i}{ }^{n}, i=1,2,3, \ldots, \mathrm{n}-1$ :

$$
\psi_{i+1}^{n+1}-\lambda \psi_{i}^{n+1}+\psi_{i-1}^{n+1}=d_{i}
$$

The related parameters can be obtained by solving the linear algebraic equations that are consisted by Eq. (21) and the boundary conditions. Without consideration of the pressure-sensitive effects, the compression factor $c$ is set as a constant:

$$
c=\frac{1}{p}
$$

\subsubsection{Establishment of mathematical model}

\section{(1) Definition of non-uniform grids}

Definition of the non-uniform grids should follow the rules that the distance further from the well is, the greater the grid is; the grid in the region near the wells is smaller. Because the distance further from the well is, the pressure decreases more slowly.

It is known from Chen (1989) that when $x$ is the length of equidistant grids $x_{i}=i \Delta x$, then $r_{i}$ is the non-uniform grid, wherein $r_{i}=r_{w} e^{x_{i}}=r_{w} e^{i \Delta x}, \frac{r_{i+1}}{r_{i}}=e^{\Delta x}$.

Next, $x_{i}: r_{i}=r_{w} e^{i \Delta x}, i=0,1,2,3, \ldots, \mathrm{n}$.

\section{(2) Tridiagonal coefficient matrix equation}

$$
\left.\frac{\partial \psi}{\partial r}\right|_{r=r_{e}}=0
$$


Combining Eqs. (21) and (23), we have:

$$
\psi_{n-1}^{n+1}-\lambda \psi_{n}^{n+1}+\psi_{n+1}^{n+1}=d_{n}
$$

It is known from $\left.\frac{2 \pi K h r}{\mu} \frac{\partial \psi}{\partial x}\right|_{x=0}=q$ that:

$$
\frac{\psi_{1}-\psi_{0}}{\Delta x}=\frac{\mu q}{86.4 \times 2 \pi K h}, \psi_{0}=\psi_{w f}
$$

Thus:

$$
\begin{gathered}
d_{0}=\frac{\mu q}{86.4 \times 2 \pi K h} \Delta x \\
-\psi_{w f}+\psi_{1}=d_{0}
\end{gathered}
$$

For points $i=1,2,3, \ldots, \mathrm{n}-2$, the tridiagonal coefficient matrix equation could be calculated as:

$$
\begin{gathered}
i=0 \\
i=1 \\
i=2 \\
\vdots \\
i=n-1 \\
i=n
\end{gathered}\left[\begin{array}{cccccc}
-1 & 1 & & & & \\
-1 & -\lambda & 1 & & & \\
& 1 & -\lambda & 1 & & \\
& & \vdots & \vdots & \vdots & \\
& & & 1 & -\lambda & 1 \\
& & & & 2 & -\lambda
\end{array}\right]\left[\begin{array}{c}
\psi_{w f} \\
\psi_{1} \\
\psi_{2} \\
\vdots \\
\psi_{n-1} \\
\psi_{n}
\end{array}\right]=\left[\begin{array}{c}
d_{0} \\
d_{1} \\
d_{2} \\
\vdots \\
d_{n-1} \\
d_{n} \\
(27)
\end{array}\right.
$$

In this paper, the mathematical model from Eq. (2) with consideration of the pressure-sensitive effects is called the Roberts P-S model; the mathematical model from Eq. (3) with consideration of the pressure-sensitive effects is called the HU P-S model; the mathematical model from Eq. (2) without consideration of the pressure-sensitive effects is called the Roberts model; the mathematical model from Eq. (3) without consideration of the pressure-sensitive effects is called the HU model.

Finally, the Gauss iterative method is used to solve the tridiagonal coefficient matrix equation.

\section{Evaluation of reservoir pressure-sensitive ef- fects}

Pressure-sensitive effects refer to the permeability of reservoirs and other physical parameters decline with the pressure decrease. In gas reservoirs with heavy pressure-sensitive effects, a suitable production pressure difference is of great significance to stabilize production, improve recovery, and extend the production time. In recent years, many experiments have been conducted in low permeability reservoirs to understand the issues. Wang et al. (2009) showed that there are heavy pressure-sensitive effects in low-permeability reservoirs; permeability reduces rapidly with the pressure decrease. Thus, it is harder to produce more gas. Furthermore, the experiments illustrated that the reservoirs destruction is irreversible. According to the conclusion above, low permeability reservoirs are not suitable for producing under a large pressure difference.

The equation used to describe the relationship between permeability under different pressures and the initial permeability can be obtained by the experiments in Wang et al.
(2015):

$$
\frac{K_{i}}{K}=e^{-\alpha\left(p_{e}-p_{i}\right)}
$$

where $K$ is the initial permeability, $\mathrm{D} ; K_{i}$ is the current penetration, $\mathrm{D} ; p_{e}$ is the original formation pressure, MPa; $p_{i}$ is the current formation pressure, $\mathrm{MPa}$; and $\alpha$ is the pressuresensitive factor.

\section{Cases calculation and analysis}

\subsection{Case 1}

Ru-1 well is a typical high-sulfur gas well. The basic information is: $\mathrm{H}_{2} \mathrm{~S}$ content: $7.3 \%$ (the second type of parameters in $\mathrm{Hu}$ et al. (2014)); gas reservoir radius: $200 \mathrm{~m}$; wellbore radius: $0.1 \mathrm{~m}$; initial permeability $3 \mathrm{mD}$; initial porosity 0.062 ; fluid viscosity: $0.0252 \mathrm{mPa} \cdot \mathrm{s}$; production: $580,000 \mathrm{~m}^{3} / \mathrm{d}$; original formation pressure: $44 \mathrm{MPa}$; bottom-hole temperature: 375.6 $\mathrm{K}$; solid sulfur density: $2.63 \mathrm{~g} / \mathrm{cm}^{3}$; relative density: 0.72 ; effective height: $3 \mathrm{~m}$.

As shown in Fig. 1, both in Roberts and HU models, the pressure reduces rapidly in a very short period of time after the gas well goes into operation. And then, the pressure decreases slowly gradually. From the curves of the Roberts model and the HU model, it is easily found that the pressure drop is approximately $20 \mathrm{MPa}$ in the Roberts model, and the pressure drop is approximately $4 \mathrm{MPa}$ in the HU model after 8 years. This figure illustrates that the predicted impact of sulfur deposition is more serious in Roberts model than in the HU model.

As shown in Fig. 2, in both models, the permeability reduces rapidly in a very short period of time after the gas well goes into operation; and it decreases slowly gradually. It can be easily found that after 8 years, the permeability drop is approximately $2 \mathrm{mD}$ in the Roberts model and it is roughly $0.02 \mathrm{mD}$ in the HU model, which is much smaller than the permeability drop in the Roberts model. This figure illustrates that the predicted reservoir damage is much more serious in the Roberts model than in the HU model.

It could be found in Fig. 3 that both in the Roberts P-S model and the HU P-S model, the pressure reduces rapidly at

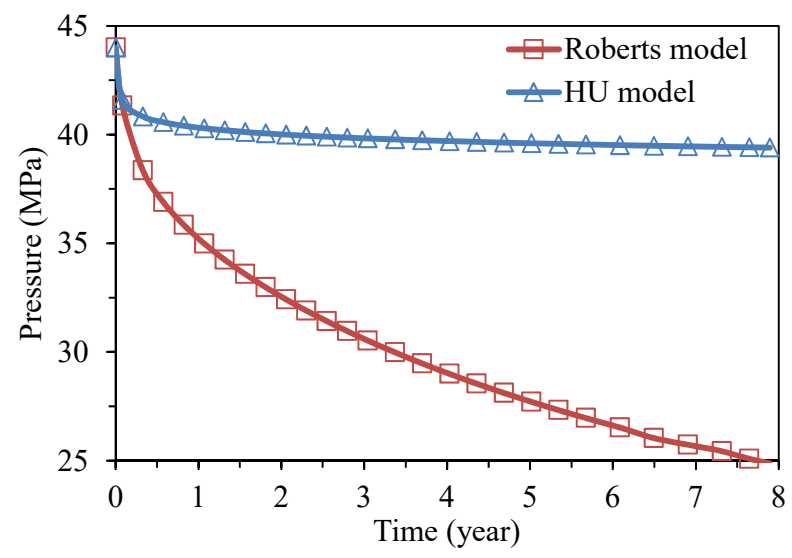

Fig. 1. Relationship between the pressure in Mesh 1 and time in the different models. 


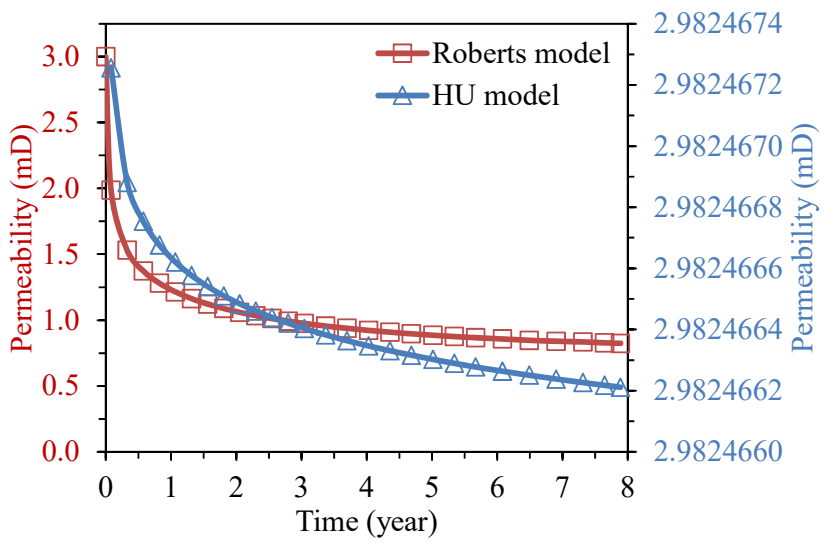

Fig. 2. Relationship between the permeability in Mesh 1 and time in the different models.

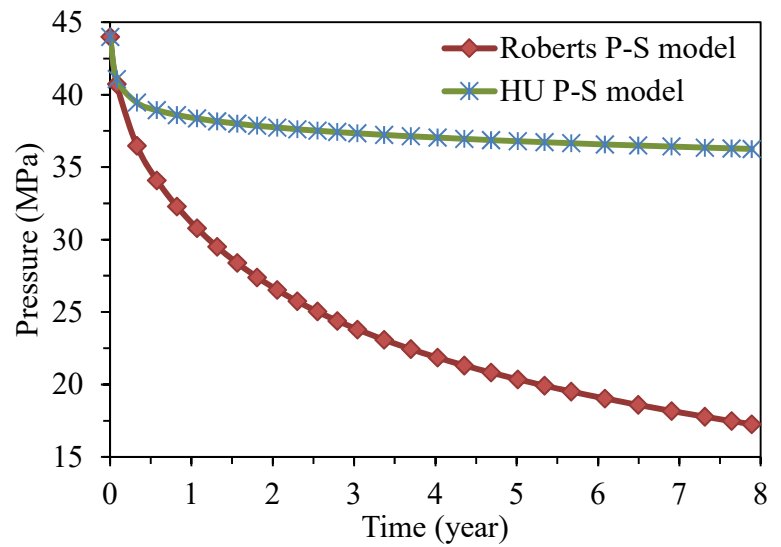

Fig. 3. Relationship between the pressure in Mesh 1 and time in the different models.

the short period of time after the gas well goes into operation; and then, the decreasing range gradually decreases. Compared with Fig. 1, the pressure is lower with consideration of the pressure-sensitive effects. It could be found that the pressure drop is approximately $28 \mathrm{MPa}$ in the Roberts P-S model after 8 years, and it is roughly $10 \mathrm{MPa}$ in HU P-S model. The pressure drop in the Roberts P-S model is considerably larger than in the HU P-S model. This figure illustrates that the predicted impact of sulfur deposition is more serious in Roberts P-S model than in the HU P-S model, and the impact will be more serious with consideration of the pressure-sensitive effects.

It could be observed in Fig. 4 that both in the Roberts P-S model and the HU P-S model, the permeability reduces rapidly at the beginning time; and then, it decreases much more slowly gradually. It could be found clearly that the permeability drop is $2.8 \mathrm{mD}$ in the Roberts P-S model and roughly $0.8 \mathrm{mD}$ in the HU P-S model after 8 years. Fig. 4 illustrates that the predicted reservoir damage is much more serious in the Roberts P-S model than in the HU P-S model, and compared with Fig. 2 , the damage will be more serious with consideration of the pressure-sensitive effects.

As shown in Fig. 5, the relationship between the pressure

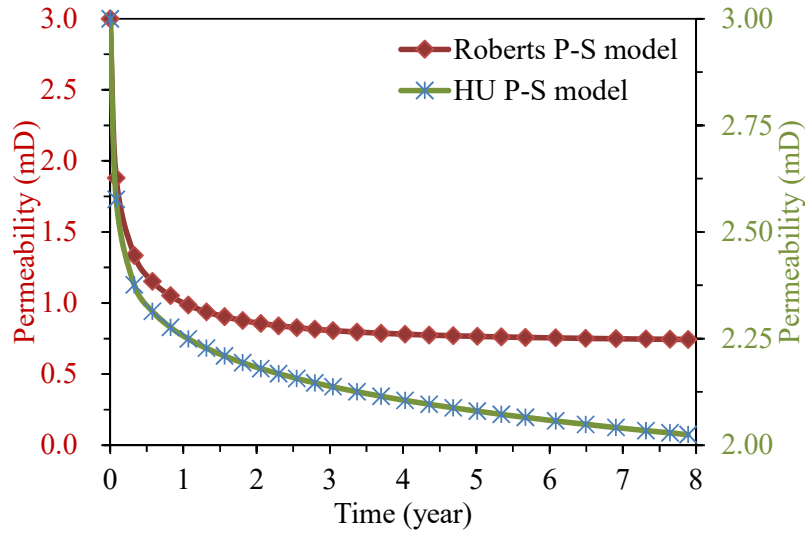

Fig. 4. Relationship between the permeability in Mesh 1 and time in the different models.

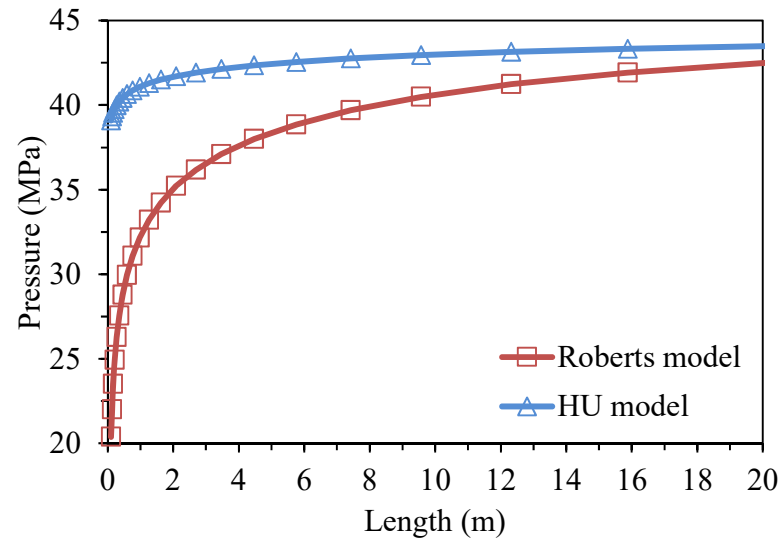

Fig. 5. Relationship between pressure and length in the different models.

and the length at the test point in the radial direction. In both models, the pressure reduces rapidly in the area which is near to the wellbore; the pressure decreases slowly further from the wellbore. The biggest pressure drop is approximately 24 $\mathrm{MPa}$ in the Roberts model; and it is roughly $8 \mathrm{MPa}$ in the HU model. The pressure drop in the Roberts model is much greater than it in the HU model. This figure illustrates that the predicted impact of sulfur deposition is more serious in the region near the wellbore, and at the same point, the predicted impact of sulfur deposition is more serious in the Roberts model than in the HU model.

As shown in Fig. 6, in both models, the permeability reduces sharply in the range of 3 meters or less from the well. It can be obtained easily from Fig. 6 that the biggest permeability drop is approximately $2.3 \mathrm{mD}$ in the Roberts model and $0.02 \mathrm{mD}$ in the $\mathrm{HU}$ model. Reservoir damage predicted by the Roberts model is much more serious than by the HU model.

Fig. 7 shows the relationship between the pressure and the length. As observed in both models, the pressure reduces rapidly in the region which is near to the wellbore; and then the pressure decreases slowly further from the wellbore. The biggest pressure drop is $28 \mathrm{MPa}$ in the Roberts P-S model and 


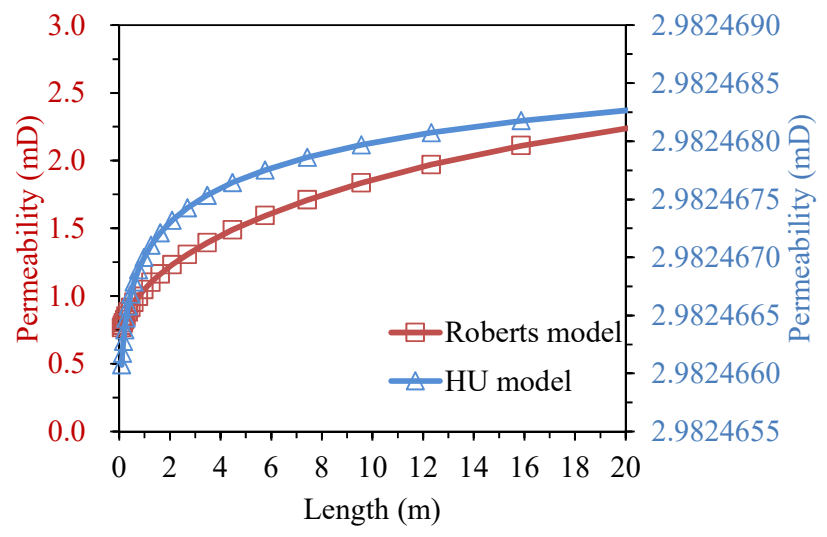

Fig. 6. Relationship between permeability and length in the different models.

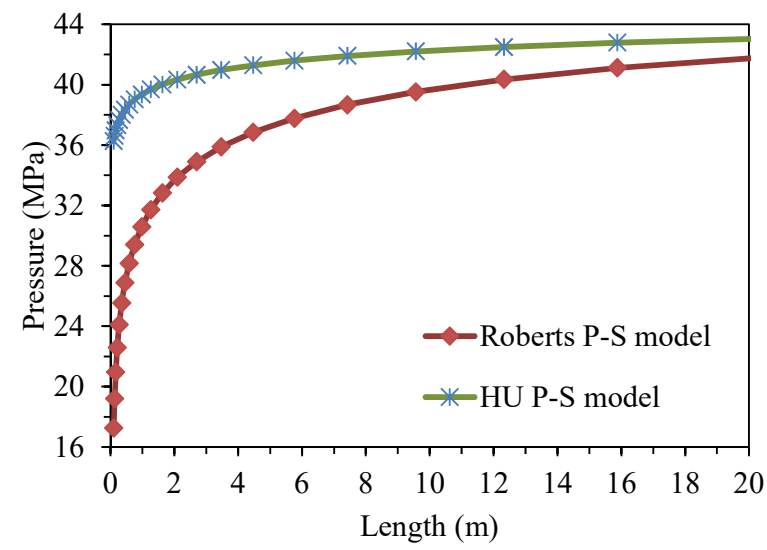

Fig. 7. Relationship between pressure and length in the different models.

it is $8 \mathrm{MPa}$ in the HU P-S model. Therefore, the Roberts P$\mathrm{S}$ model has a more serious impact to high-sulfur gas well development. Fig. 7 illustrates that the predicted impact of sulfur deposition is much more serious in the Roberts P-S model than in the HU P-S model, and the impact will be more serious with consideration of the pressure-sensitive effects.

As shown in Fig. 8, in both models, the permeability reduces sharply in the range of 3 meters or less of the wellbore, and then it decreases slowly further from the wellbore. It could be found that the biggest permeability drop is $2.3 \mathrm{mD}$ in the Roberts P-S model and $0.2 \mathrm{mD}$ in the HU P-S model. Reservoir damage calculated by the Roberts P-S model is more serious than by the HU P-S model. Fig. 8 illustrates that the predicted reservoir damage is much more serious in the Roberts P-S model than in the HU P-S model, and the damage will be more serious with consideration of the pressure-sensitive effects.

\subsection{Case 2}

$\mathrm{Ru}-2$ well is a typical high-sulfur gas well. Basic information about the well is: $\mathrm{H}_{2} \mathrm{~S}$ content: $5.8 \%$ (the third type of parameters in $\mathrm{Hu}$ et al. (2014)); gas reservoir radius: $200 \mathrm{~m}$; wellbore radius: $0.1 \mathrm{~m}$; initial permeability $3 \mathrm{mD}$; initial poro-

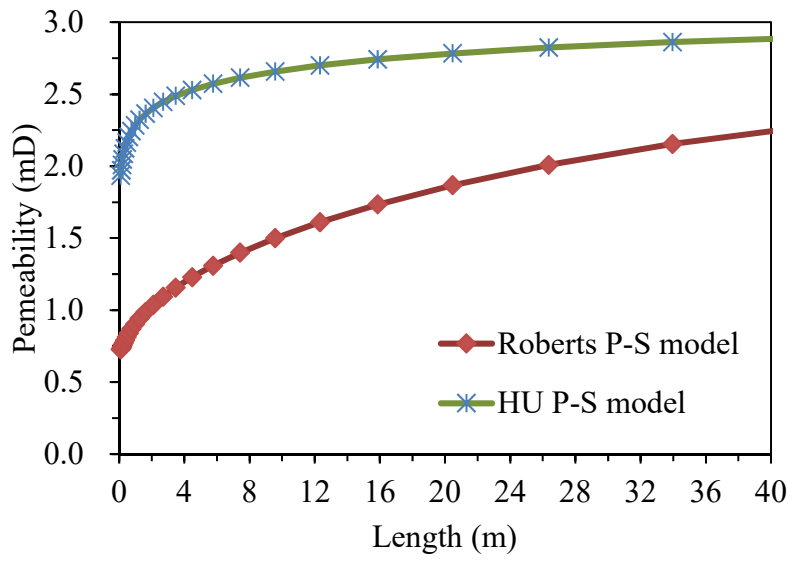

Fig. 8. Relationship between permeability and length in the different models.

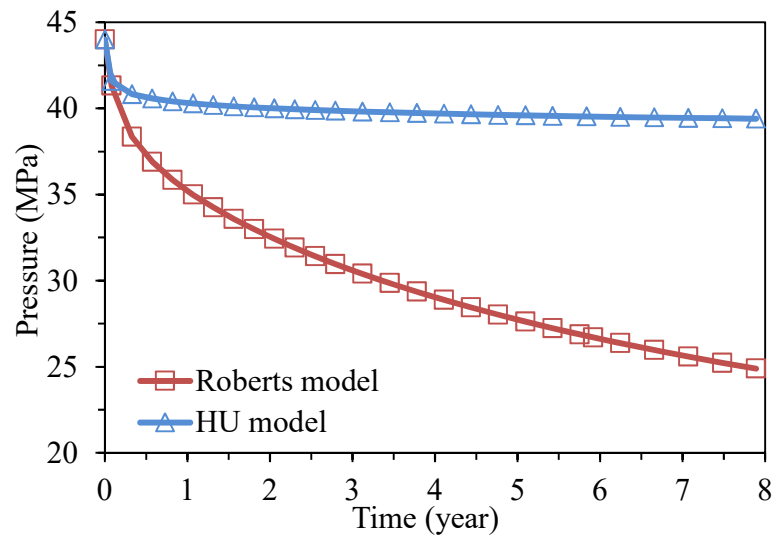

Fig. 9. Relationship between pressure in 1st Mesh and time in the different models.

sity 0.062 ; fluid viscosity: $0.0252 \mathrm{mPa} \cdot \mathrm{s}$; production: 580,000 $\mathrm{m}^{3} / \mathrm{d}$; original formation pressure: $44 \mathrm{MPa}$; bottom-hole temperature: $375.6 \mathrm{~K}$; solid sulfur density: $2.63 \mathrm{~g} / \mathrm{cm}^{3}$; relative density: 0.72; effective height: $3 \mathrm{~m}$.

As shown in Fig. 9, both in Roberts and HU models, the pressure reduces rapidly in a very short period of time after the gas well goes into operation. And then, the pressure decreases slowly gradually. From the curves of the Roberts model and the HU model, it is easily found that the pressure drop is approximately $20 \mathrm{MPa}$ in the Roberts model, and the pressure drop is approximately $4 \mathrm{MPa}$ in the $\mathrm{HU}$ model after 8 years. This figure illustrates that the predicted impact of sulfur deposition is more serious in Roberts model than in the HU model.

As shown in Fig. 10, in both models, the permeability reduces rapidly in a very short period of time after the gas well goes into operation; and it decreases slowly gradually. It can be easily found that after 8 years, the permeability drop is approximately $2.6 \mathrm{mD}$ in the Roberts model and it is roughly $0.02 \mathrm{mD}$ in the $\mathrm{HU}$ model, which is much smaller than the permeability drop in the Roberts model. This figure illustrates that the predicted reservoir damage is much more serious in the Roberts model than in the HU model. 


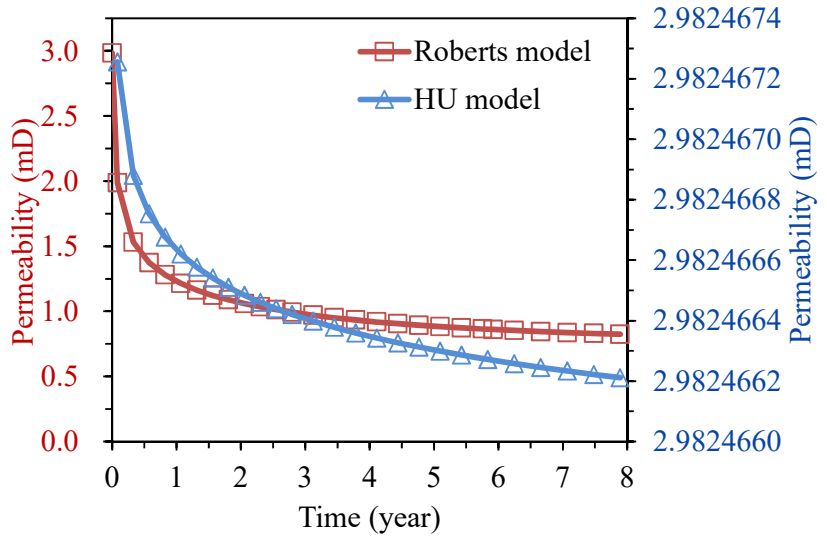

Fig. 10. Relationship between permeability in 1 st Mesh and time in the different models.

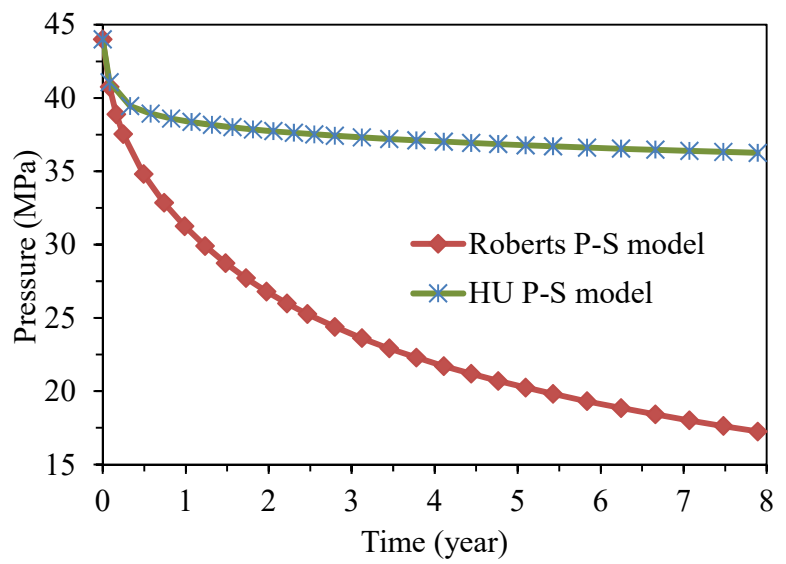

Fig. 11. Relationship between pressure in 1st Mesh and time in the different models.

It could be found in Fig. 11 that both in the Roberts P-S model and the HU P-S model, the pressure reduces rapidly at the short period of time after the gas well goes into operation; and then, the decreasing range gradually decreases. Compared with Fig. 9, the pressure is lower with consideration of the pressure-sensitive effects. It could be found that the pressure drop is approximately $27 \mathrm{MPa}$ in the Roberts P-S model after 8 years, and it is roughly $7 \mathrm{MPa}$ in HU P-S model. The pressure drop in the Roberts P-S model is considerably larger than in the HU P-S model. This figure illustrates that the predicted impact of sulfur deposition is more serious in Roberts P-S model than in the HU P-S model, and the impact will be more serious with consideration of the pressure-sensitive effects.

It could be observed in Fig. 12 that both in the Roberts P-S model and the HU P-S model, the permeability reduces rapidly at the beginning time; and then, it decreases much more slowly gradually. It could be found clearly that the permeability drop is $2.75 \mathrm{mD}$ in the Roberts P-S model and roughly $1 \mathrm{mD}$ in the HU P-S model after 8 years. Fig. 12 illustrates that the predicted reservoir damage is much more serious in the Roberts P-S model than in the HU P-S model, and compared with Fig. 10, the damage will be more serious with considera-

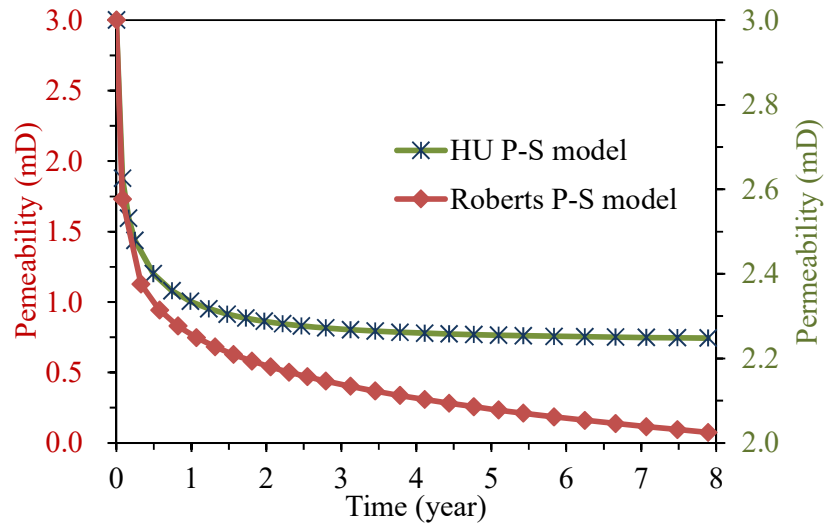

Fig. 12. Relationship between permeability in 1st Mesh and time in the different models.

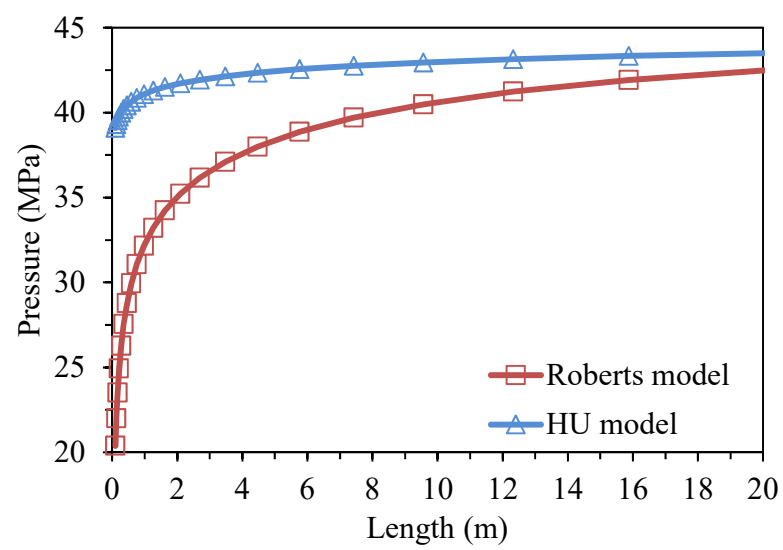

Fig. 13. Relationship between pressure and length in the different models.

tion of the pressure-sensitive effects.

As shown in Fig. 13. In both models, the pressure reduces rapidly in the area which is near to the wellbore; the pressure decreases slowly further from the wellbore. The biggest pressure drop is approximately $24 \mathrm{MPa}$ in the Roberts model; and it is roughly $6 \mathrm{MPa}$ in the HU model. The pressure drop in the Roberts model is much greater than it in the HU model. This figure illustrates that the predicted impact of sulfur deposition is more serious in the region near the wellbore, and at the same point, the predicted impact of sulfur deposition is more serious in the Roberts model than in the HU model.

As shown in Fig. 14, in both models, the permeability reduces sharply in the range of 3 meters or less from the well. It can be obtained easily from Fig. 6 that the biggest permeability drop is approximately $2.25 \mathrm{mD}$ in the Roberts model and $0.02 \mathrm{mD}$ in the $\mathrm{HU}$ model. Reservoir damage predicted by the Roberts model is much more serious than by the HU model.

Fig. 15 shows the relationship between the pressure and the length. As observed in both models, the pressure reduces rapidly in the region which is near to the wellbore; and then the pressure decreases slowly further from the wellbore. The biggest pressure drop is $28 \mathrm{MPa}$ in the Roberts P-S model and 


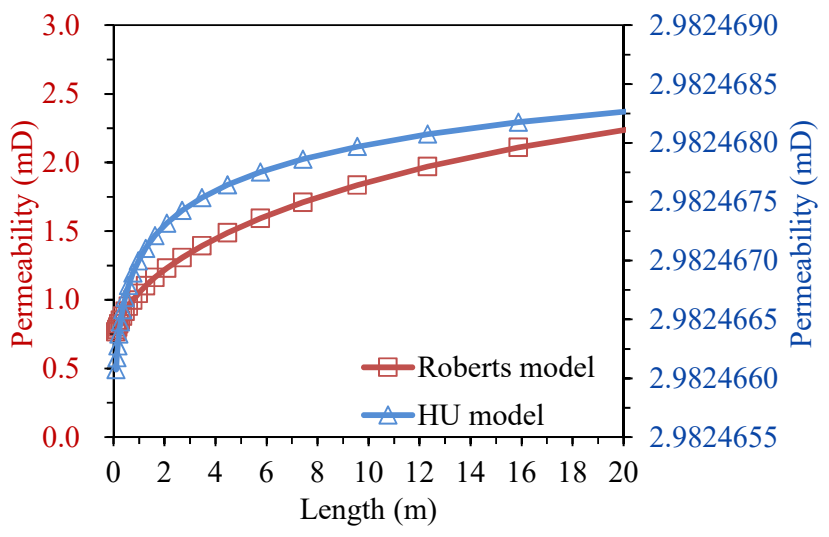

Fig. 14. Relationship between permeability and length in the different models.

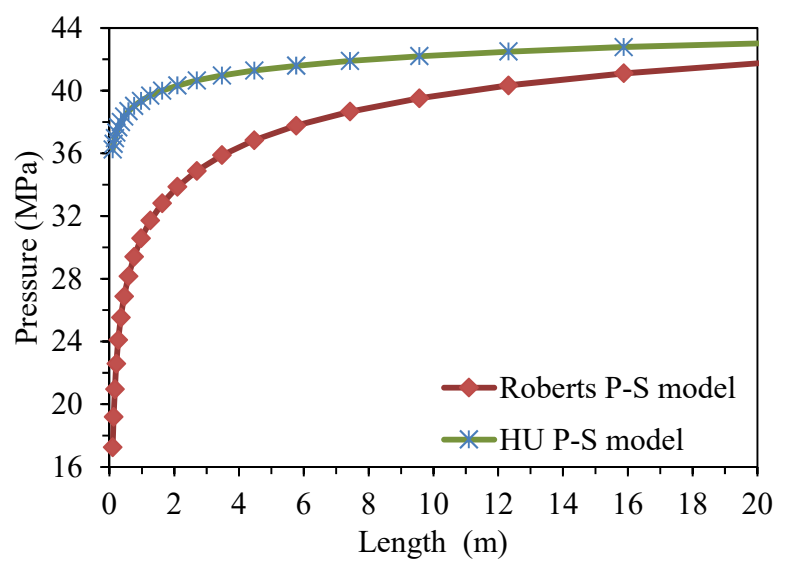

Fig. 15. Relationship between pressure and length in different models.

it is $8 \mathrm{MPa}$ in the HU P-S model. Therefore, the Roberts P$\mathrm{S}$ model has a more serious impact to high-sulfur gas well development. Fig. 15 illustrates that the predicted impact of sulfur deposition is much more serious in the Roberts P-S model than in the HU P-S model, and the impact will be more serious with consideration of the pressure-sensitive effects.

As shown in Fig. 16, in both models, the permeability reduces sharply in the range of 3 meters or less of the wellbore, and then it decreases slowly further from the wellbore. It could be found that the biggest permeability drop is $2.5 \mathrm{mD}$ in the Roberts P-S model and $1.2 \mathrm{mD}$ in the HU P-S model. Reservoir damage calculated by the Roberts P-S model is more serious than by the HU P-S model. Fig. 16 illustrates that the predicted reservoir damage is much more serious in the Roberts P-S model than in the HU P-S model, and the damage will be more serious with consideration of the pressure-sensitive effects.

\section{Conclusions}

1) A new formula is provided to describe permeability and pressure changes in high-sulfur gas reservoirs with consideration of pressure-sensitive effects and sulfur deposition.

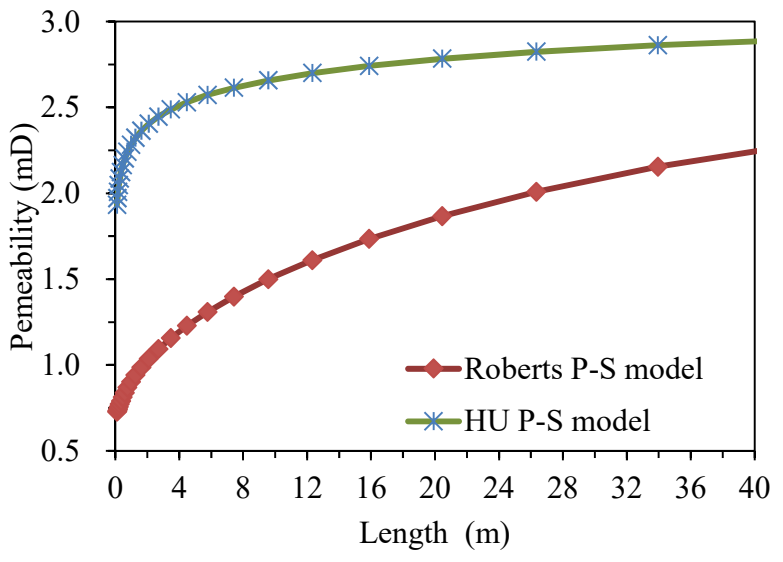

Fig. 16. Relationship between permeability and length in the different models.

2) The mathematical model of a high-sulfur gas well under the condition of a closed outer boundary and an internal boundary of fixed production has been established and solved by a Gauss iterative method.

3) It is reflected that the permeability damage caused by sulfur deposition is mainly in the range of 3 meters or less from the wellbore. When the length is shorter and the amount of solid sulfur is greater, and the pressuresensitive effects become more serious.

4) The predicted pressure drop and permeability drop are much smaller in HU model than Roberts model. The pressure drop and permeability drop will be bigger with consideration of the pressure-sensitive effects.

\section{Acknowledgments}

The authors would like to acknowledge the support provided by the National Natural Science Foundation of China (51304174), National Science and Technology Major Project (2017ZX05013002) and the Fundamental Research Funds for the Central Universities (2652018209).

Open Access This article is distributed under the terms and conditions of the Creative Commons Attribution (CC BY-NC-ND) license, which permits unrestricted use, distribution, and reproduction in any medium, provided the original work is properly cited.

\section{References}

Bian, X., Zhang, L., Du, Z., et al. Prediction of sulfur solubility in super critical sour gases using grey wolf optimizerbased support vector machine. J. Mol. Liq. 2018, 261: 1735-1760.

Bian, X., Zhang, Q., Du, Z., et al. A five-parameter empirical model for correlation the solubility of solid compounds in supercritical carbon dioxide. Fluid Phase Equilibr. 2016, 411: 74-80.

Cen, F., Lai, F., Jiang, H., et al. An improved calculation method for reserve of constant-volume sour gas reservoirs. Oil \& Gas Geology 2007, 28(3): 320-323. (in Chinese) 
Chen, Y. Reservoir simulation basis. Beijing, Petroleum Industry Press, 1989. (in Chinese)

Chrastil, J. Solubility of solids and liquids in supercritical gases. Phys. Chem. 1982, 86: 3016-3021.

Clark, P.D., Lesage, K.L., Sarkar, P. Application of aryl disulfides for the mitigation of sulfur deposition in sour gas wells. Energy Fuels 1989, 3(3): 315-320.

Fan, Z., Li, H., Liu, J., et al. The elemental sulfur deposition and its corrosion in high sulfur gas fields. Natural Gas Industry 2013, 33(9): 102-109. (in Chinese)

Guo, X., Du, Z., Yang, X., et al. Sulfur deposition in sour gas reservoirs: Laboratory and simulation study. Pet. Sci. 2009, 6(4): 405-414.

Guo, X., Wang, P., Liu, J., et al. Gas well water breakthrough time model for high sulfur gas reservoirs considering sulfur deposition. J. Pet. Sci. Eng. 2017, 157: 999-1006.

Guo, X., Wang, Q. A new prediction model of elemental sulfur solubility in sour gas mixtures. J. Nat. Gas Sci. Eng. 2016, 31: 98-107.

He, J., Rui, Z., Ling, K. A new method to determine Biot's coefficients of Bakken samples. J. Nat. Gas Sci. Eng. 2016, 35: 259-264.

Hu, J., Chong, Z., Zhe, H., et al. Fractured horizontal well productivity prediction in tight oil reservoirs. J. Pet. Sci. Eng. 2017b, 151: 159-168.

Hu, J., Lei, Z., Chen, Z., et al. Effect of sulfur deposition on well performance in a sour gas reservoir. Can. J. Chem. Eng. 2017a, 96(4): 886-894.

$\mathrm{Hu}$, J., Zhao, J., Wang, L., et al. Prediction model of elemental sulfur solubility in sour gas mixtures. J. Nat. Gas Sci. Eng. 2014, 18: 31-38.

Karan, K., Heidemann, R.A., Behie, L.A. Sulfur solubility in sour gas: Predictions with an equation of state model. Ind. Eng. Chem. Res. 1998, 37(5): 1679-1684.

Li, C., Liu, G., Peng, Y. Predicting sulfur solubility in hydrogen sulfide, carbon dioxide, and methane with an improved thermodynamic model. RSC Adv. 2018, 8: 16069-16081.

Li, Z., Gu, T., Guo, X., et al. Characterization of the unidirectional corrosion of oilwell cement exposed to $\mathrm{H}_{2} \mathrm{~S}$ under high-sulfur gas reservoir conditions. RSC Adv. 2015, 5: 71529-71536.

Mao, J., Yang, X., Wang, D. Optimization of effective sulfur solvents for sour gas reservoir. J. Nat. Gas Sci. Eng. 2016, 36: 463-471.

Ou, C., Rui, R., Li, C., et al. Multi-index and two-level evaluation of shale gas reserve quality. J. Nat. Gas Sci. Eng. 2016, 35: 1139-1145.
Pirzadeh, P., Lesage, K.L., Marriott, R.A. Hydraulic fracturing additives and the delayed onset of hydrogen sulfide in shale gas. Energy Fuels 2014, 28(8): 4993-5001.

Ren, Z., Wu, X., Liu, D., et al. Semi-analytical model of the transient pressure behavior of complex fracture networks in tight oil reservoirs. J. Nat. Gas Sci. Eng. 2016, 35: 497-508.

Roberts, B.E. The effect of sulfur deposition on gas well inflow performance. SPE Reserv. Eng. 1997, 12(2): 118123.

Roberts, B.E. Flow impairment by deposited sulfur-A review of 50 years of research. J. Nat. Gas Eng. 2017, 2(1): 84-105.

Santos, J., Lobato, A., Moraes, C., et al. Comparison of different processes for preventing deposition of elemental sulfur in natural gas pipelines: A review. J. Nat. Gas Sci. Eng. 2016, 32: 364-372.

Sun, C., Chen, G. Experimental and modeling studies on sulfur solubility in sour gas. Fluid Phase Equilibr. 2003, 214(2): 187-195.

Sun, J., Gamboa, E., Schechter, D., et al. An integrated workflow for characterization and simulation of complex fracture networks utilizing micro seismic and horizontal core data. J. Nat. Gas Sci. Eng. 2016, 34: 1347-1360.

Wang, F., Li, X., Couples, G., et al. Stress arching effect on stress sensitivity of permeability and gas well production in Sulige gas field. J. Pet. Sci. Eng. 2015, 125: 234-246.

Wang, R., Xiang, A., Zhao, R., et al. Effect of stress sensitivity on displacement efficiency in $\mathrm{CO}_{2}$ flooding for fractured low permeability reservoirs. Pet. Sci. 2009, 6(3): 277-283.

Wang, S. The relationship between sulfur deposition and gas production capacity. Pet. Explor. Dev. 1999, 5: 56-58.

Wang, W., Liu, C., Mu, L., et al. Technical policy optimization for the development of carbonate sour gas reservoirs. Oil \& Gas Geology 2011, 32(2): 302-310. (in Chinese)

Yang, C. Natural gas production engineering. Beijing, Petroleum Industry Press, 2001. (in Chinese)

Zeng, D., Peng, X., Fu, D., et al. Development dynamic monitoring technologies used in the Puguang high-sulfur gas field. Natural Gas Industry 2019, 6(3): 191-197. (in Chinese)

Zeng, P., Zhao, J., Zhou, H. Formation damage from elemental sulfur deposition in sour gas reservoir. Pet. Explor. Dev. 2005, 32(6): 113-115.

Zhao, X., Rui, Z., Liao, X., et al. A simulation method for modified isochronal well testing to determine shale gas well productivity. J. Nat. Gas Sci. Eng. 2015, 27(2): 479485 . 